\title{
Submental Lymph Node Group (Sublevel
}

IA)

National Cancer Institute

\section{Source}

National Cancer Institute. Submental Lymph Node Group (Sublevel IA). NCI Thesaurus.

Code C132508.

Lymph nodes within the triangular boundary of the anterior belly of the digastric muscles and the hyoid bone. These nodes are at greatest risk for harboring metastases from cancers arising from the floor of mouth, anterior oral tongue, anterior mandibular alveolar ridge, and lower lip. (AJCC 8th ed.) 